\title{
Primary peritoneal mesothelioma: A rare cause of malignant ascites
}

\author{
Joseph W Howells ${ }^{1}$, Bryan J Kantner ${ }^{2}$, J oel T Abbott ${ }^{1}$ \\ 1. Department of Medicine, Madigan Army Medical Center, Tacoma, WA, USA. 2. Department of Radiology, Madigan Army \\ Medical Center, Tacoma, WA, USA
}

Correspondence: Joseph W Howells. Address: 7142 Axis St SE, Lacey, WA 98513, USA.

E-mail: joseph.w.howells.mil@mail.mil

Received: August 6, 2015

DOI : $10.5430 /$ crim.v2n4p17
Accepted: August 27, 2015

URL: http://dx.doi.org/10.5430/crim.v2n4p17
Online Published: September 7, 2015

\section{Abstract}

Introduction: The evaluation of peritoneal masses requires a focused, systematic approach. While peritoneal fluid analysis and axial imaging are essential to the workup, further analysis is often needed for a final diagnosis. We present a case of primary peritoneal mesothelioma mimicking peritoneal carcinomatosis of gastrointestinal origin on computerized tomography (CT) imaging.

Case presentation: A 61-year-old man presented to the hospital with an approximate 1-month history of abdominal distention, dyspnea, nausea and fatigue. Peritoneal studies revealed serum-ascites-albumin-gradient (SAAG) of less than 1.1, consistent with non-portal hypertension ascites. CT abdomen revealed large-volume ascites with omental caking, concerning for peritoneal carcinomatosis. Peritoneal fluid cytology was initially inconclusive, prompting ultrasound-guided peritoneal biopsies, which revealed primary abdominal mesothelioma.

Conclusions: Neither CT nor peritoneal cytology are typically sufficient for the diagnosis of peritoneal mesothelioma. When strong clinical suspicion exists, early tissue biopsies should be pursued.

\section{Keywords}

Peritoneal, Mesothelioma, Ascites, Carcinomatosis

\section{I ntroduction}

Primary peritoneal mesothelioma is a rare and often overlooked cause of ascites. It represents approximately $30 \%$ of all mesotheliomas ${ }^{[1]}$, with an overall incidence of approximately 250 cases per year in the United States ${ }^{[2]}$. As with pleural mesothelioma, it has been linked to asbestos exposure, likely gaining access to the abdominal cavity from inhalation followed by coughing and swallowing ${ }^{[2]}$. The clinical presentation of peritoneal mesothelioma is highly variable, with abdominal distention, pain and weight loss as the most common initial symptoms ${ }^{[3]}$.

Prompt diagnosis of peritoneal mesothelioma is essential, as there are emerging surgical therapies which have produced highly encouraging results ${ }^{[4]}$. As this case demonstrates, CT findings can be nonspecific and peritoneal cytology is often inconclusive, underscoring the importance of early tissue sampling for diagnosis. 


\section{Case presentation}

A 61-year-old man presented to the hospital with an approximate one month history of abdominal distention, difficulty breathing, nausea and fatigue. His medical history was notable for chronic hepatitis $C$, treated with antiviral therapy five years prior, with sustained virologic response. He was a former smoker, with a 15-pack-year history, and drank one 2-oz glass of whiskey daily (recently quit one month prior). He formerly worked as an aviation mechanic in the military.

The patient had been admitted to an outside facility 1 week prior, where a paracentesis revealed lymphocyte-predominant ascitic fluid (808 wbc/cmm, 96\% lymphocytes, 4\% neutrophils), with a serum-ascites albumin gradient (SAAG) of less than 1.1. Gram stain and cultures were negative, cytology was inconclusive. Right upper quadrant ultrasound revealed hepatic steatosis with no focal lesions, and otherwise normal contour and portal venous flow. The patient was discharged on furosemide and spironolactone. Despite taking the diuretics as prescribed, his abdominal swelling persisted, prompting him to come to our facility for evaluation.

The patient's vital signs were within normal limits upon presentation. Examination revealed marked abdominal distention with positive fluid wave. No jaundice or other signs of liver disease were appreciated.

Laboratory studies were notable for white blood cell count of $17.4 \times 10^{3}$ cells/mm, blood urea nitrogen of $32 \mathrm{mg} / \mathrm{dl}$, and creatinine of $1.4 \mathrm{mg} / \mathrm{dl}$ (improved from $1.8 \mathrm{mg} / \mathrm{dl}$ one week prior). Hepatic panel revealed mildly elevated alanine aminotransferase (ALT) of $44 \mathrm{U} / \mathrm{L}$, and was otherwise within normal limits. Coagulation studies revealed international normalized ratio (INR) of 1.2. Peritoneal fluid studies on admission remained unchanged from those performed one week prior and cytology was without malignant cells.

CT of the abdomen with IV and oral contrast was performed (see Figure 1), which revealed large-volume ascites with omental caking, concerning for peritoneal carcinomatosis with no identified primary source.
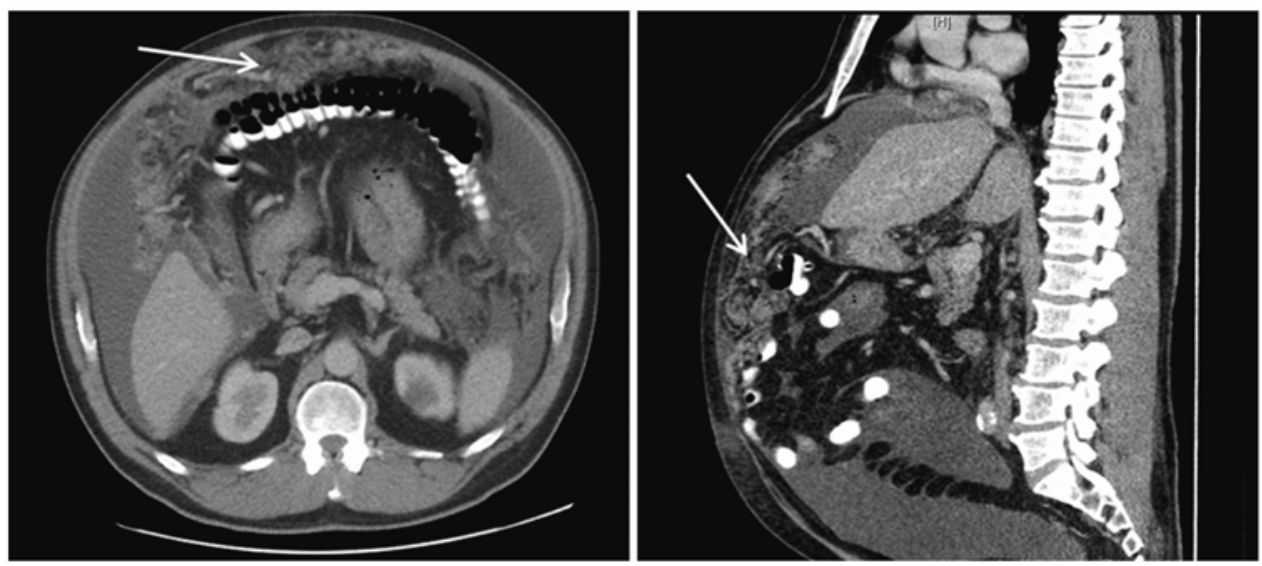

Figure 1. CT abdomen with oral and intravenous contrast showing a diffuse metastatic process with malignant ascites and omental caking (Arrows)

Tumor markers carcinoembryonic antigen (CEA), alpha-fetoprotein (AFP), prostate-specific antigen (PSA), and cancer-antigen 19-9 (CA 19-9) were all within normal limits. Contrasted CT of the chest was negative for significant thoracic pathology.

Repeat peritoneal cytology revealed malignant epitheliod tumor cells with enlarged nuclei and prominent nucleoli, concerning for mesothelioma vs. metastatic malignancy (see Figure 2). Ultrasound-guided core biopsies were then obtained, which revealed direct invasion of neoplastic and inflammatory cells into the surrounding adipose tissue (see Figure 3), with positivity for CK5/6, CK7, calretinin, D2-40, and PAX8, and negativity for CK20, CEA, CD15, and TTF-1, an immunophenotype consistent with the diagnosis peritoneal mesothelioma, epithelioid type. 

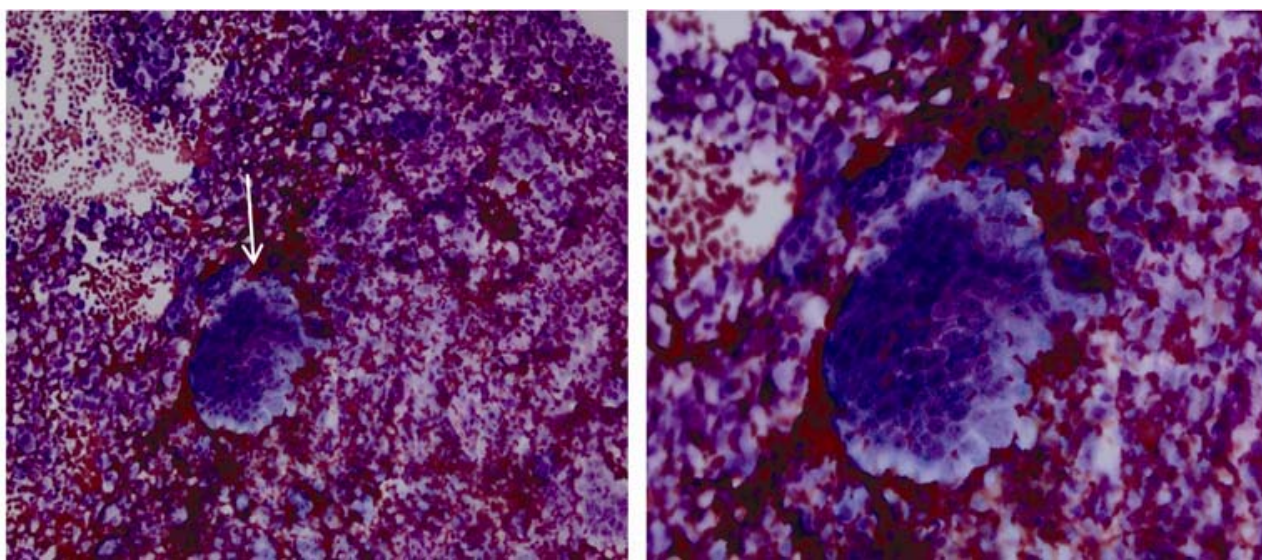

Figure 2. Cytology (DiffQuik): Medium power view (20×, left) reveals highly atypical cellularity, comprised predominantly of malignant epithelioid cells in large groups (arrow) and as single, discohesive cells. High power view (40×, right) shows large tumor cells with abundant cytoplasm, enlarged nuclei, and prominent nucleoli. Mitotic activity is rare and not readily evident
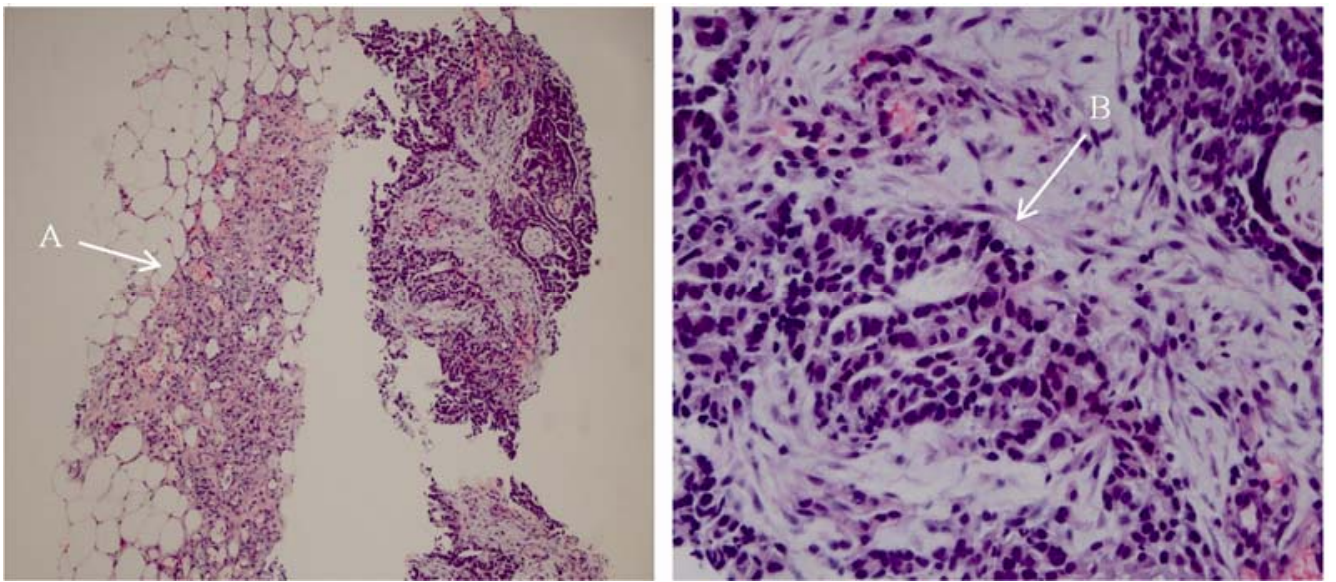

Figure 3. Needle core biopsy (HE Stain). Low power view (10×, left) reveals benign-appearing adipose tissue infiltrated by neoplastic and mixed inflammatory cells (Arrow A). High power view (40×, right) reveals poorly differentiated tumor cells in a papillary architectural formation (Arrow B)

The patient was referred to oncology for consideration of treatment options. Unfortunately, his disease continued to rapidly progress (see Figure 4), resulting in a sharp decline in his functional status and renal function. He was deemed to be an unsuitable candidate for palliative chemotherapy or surgery, and died less than 2 months after diagnosis.
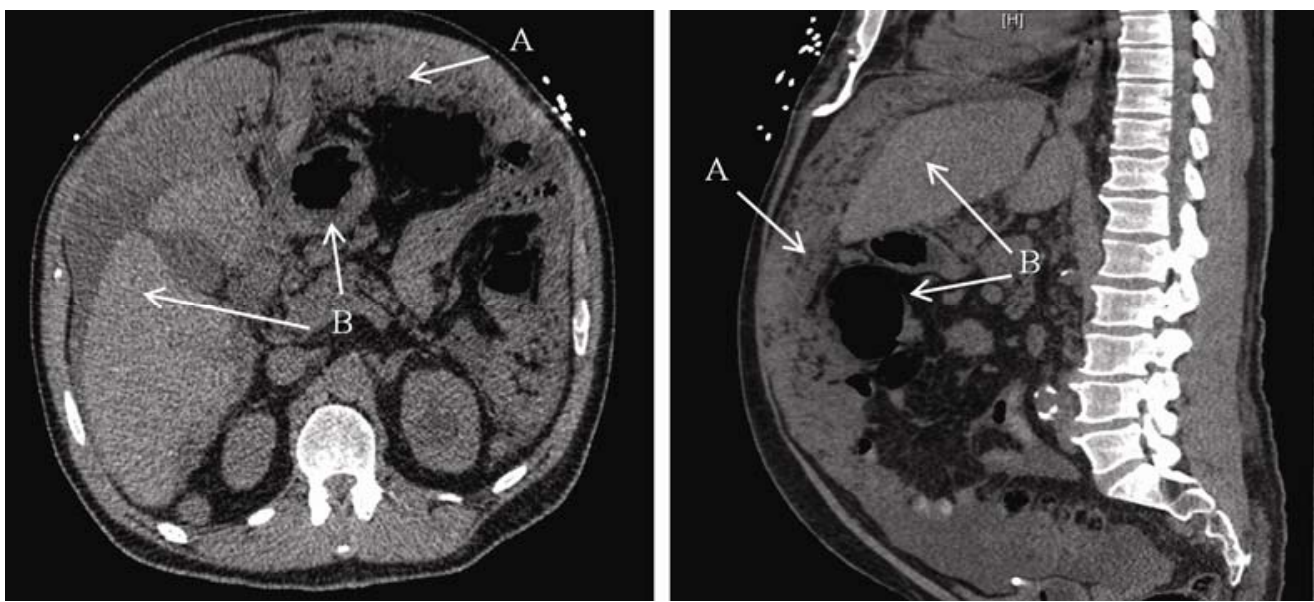

Figure 4. CT Abdomen without contrast 26 days later: axial (Left) and sagittal (Right) views showing interval progression of peritoneal malignancy, with increasing bulk and density (A) and re-demonstrated mass effect as seen with the shift of liver and bowel (B) 


\section{Discussion}

This case highlights the importance of maintaining a broad differential diagnosis when evaluating new-onset ascites with peritoneal masses. Given the patient's history of hepatitis $C$ and lack of recent colorectal cancer screening, the initial concern in this case was for peritoneal carcinomatosis, likely of gastrointestinal origin. However, negative tumor markers made this less likely, prompting us to expand our differential to less common etiologies.

Although a rare cause of malignant ascites, there were some historical features that made the diagnosis of peritoneal mesothelioma more favorable in this case. First, the patient was a retired aircraft mechanic, a profession which placed him at risk for asbestos exposure ${ }^{[5]}$. The rapid nature of his symptoms was also significant, as it favored a highly aggressive disease such as mesothelioma, as opposed to a more indolent metastatic process.

On CT, peritoneal mesothelioma typically presents as diffuse (more common) or localized nodular peritoneal thickening, usually accompanied with omental caking and ascites ${ }^{[6]}$. Although CT is considered a first-line imaging modality, it is fairly non-specific, as many disease processes may mimic peritoneal mesothelioma, such as peritoneal carcinomatosis from other primary malignancies, lymphoma, gastrointestinal stromal tumors (GIST), and peritoneal tuberculosis ${ }^{\text {[7] }}$.

Given the limited specificity of CT for peritoneal mesothelioma, it is essential to obtain a tissue sample whenever possible to confirm the diagnosis. Cytology of peritoneal fluid is typically low yield with a reported sensitivity ranging from $32 \%$ to $76 \%{ }^{[8]}$. This is in sharp contrast to peritoneal carcinomatosis from gastrointestinal tumors, where sensitivity of peritoneal cytology can reach up to $96.7 \%$ with serial samples ${ }^{[9]}$.

Another pitfall of peritoneal cytology is that it does not allow for visualization of tumor invasion into the surrounding tissues, an important histological feature of malignant mesothelioma. As a consequence, many pathologists are reluctant to make the diagnosis of peritoneal mesothelioma based solely on fluid cytology, as malignant mesothelial cells may often appear morphologically similar to reactive mesothelial cells from inflammatory processes, such as infection, collagen vascular disease, and surgery ${ }^{[8]}$.

Peritoneal mesothelioma is an aggressive disease that has a poor prognosis. Without treatment, patients have a median survival time of approximately 6 months ${ }^{[10]}$. For non-surgical candidates, chemotherapy regimens with pemetrexed plus platinum-based chemotherapy may extend survival times to a median of 1 year ${ }^{[10]}$. For surgical candidates, cytoreduction with hyperthermic intraperitoneal chemotherapy has produced highly encouraging results, improving median survival times to between 29.5 and 100 months ${ }^{[11]}$. Surgical cytoreduction is considered high risk, with an incidence of significant surgical complications reported in $28 \%-41 \%$ of cases ${ }^{[11]}$. This highlights the importance of prompt diagnosis and referral, both to optimize patient functional status and minimize tumor burden before surgical intervention.

\section{Conclusion}

Peritoneal mesothelioma may be indistinguishable from other causes of peritoneal carcinomatosis based on CT imaging characteristics alone ${ }^{[7]}$. When clinical concern for peritoneal mesothelioma exists, direct tissue biopsies should be pursued as early as possible to establish a diagnosis

\section{Disclaimer}

The views expressed are those of the authors and do not reflect the official policy of the Department of the Army, the Department of Defense or the U.S. Government.

\section{Acknowledgements}

Salvatore Mignano, $\mathrm{DO}^{1}$

Christina Schofield, $\mathrm{MD}^{2}$ 
1. Department of Pathology, Madigan Army Medical Center, Tacoma, WA

2. Department of Medicine, Madigan Army Medical Center, Tacoma, WA

\section{References}

[1] De Pangher Manzini V, Recchia L, Cafferata M, et al. Malignant peritoneal mesothelioma: a multicenter study on 81 cases. Ann Oncol. 2010; 21(2): 348-53. http://dx.doi.org/10.1093/annonc/mdp307.

[2] Chua TC, Chong CH., Morris DL. Peritoneal mesothelioma: current status and future directions. Surg Oncol Clin N Am. 2012; 21(4): 635-43. http://dx.doi.org/10.1016/j.soc.2012.07.010.

[3] Boffetta P. Epidemiology of peritoneal mesothelioma: a review. Ann Oncol. 2007; 18(6): 985-90. http://dx.doi.org/10.1093/annonc/mdl345.

[4] Alexander HR Jr, Bartlett DL, Pingpank JF, et al. Treatment factors associated with long-term survival following cytoreductive surgery and regional chemotherapy for patients with malignant peritoneal mesothelioma. Surgery. 2013; 153(6): 779-86. http://dx.doi.org/10.1016/j.surg.2013.01.001.

[5] O’Reilly KM, McLaughlin AM, Beckett WS. Asbestos-related lung disease. Am Fam Physician. 2007; 75(5): 683-88. PMid:17375514

[6] Vicens RA, Patnana M, Le O, et al. Multimodality imaging of common and uncommon peritoneal diseases: a review for radiologists. Abdom Imaging. 2015; 40(2): 436-56. http://dx.doi.org/10.1007/s00261-014-0224-8.

[7] Smiti S, Rajagopal KV. CT mimics of peritoneal carcinomatosis. Indian J Radiol Imaging. 2010; 20(1): 58-62. http://dx.doi.org/10.4103/0971-3026.59757.

[8] Husain AN, Colby T, Ordonez N, et al. Guidelines for pathologic diagnosis of malignant mesothelioma: 2012 update of the consensus statement from the international mesothelioma interest group. Arch Pathol Lab Med. 2013; 137(5): 647-67. http://dx.doi.org/10.5858/arpa.2012-0214-OA).

[9] Hou W, Sanyal AJ. Ascites: diagnosis and management. Med Clin N Am. 2009; 93(4): 801-17. http://dx.doi.org/10.1016/j.mcna.2009.03.007.

[10] Turner K, Varghese S, Alexander HR Jr. Current concepts in the evaluation and treatment of patients with diffuse malignant peritoneal mesothelioma. J Natl Compr Canc Netw. 2012; 10(1): 49-57. PMid:22223869

[11] Raza A, Huang WC, Takabe K. Advances in the management of peritoneal mesothelioma. World J Gastroenterol. 2014; 20(33): 11700-12. http://dx.doi.org/10.3748/wjg.v20.i33.11700 